\title{
Characterization of Perineum Elasticity and Pubic Bone-Perineal Critical Distance with a Novel Tactile Probe: Results of an Intraobserver Reproducibility Study
}

\author{
Justin S. Brandt'1, Todd Rosen¹, Heather Van Raalte², Viktors Kurtenos ${ }^{3}$, Vladimir Egorov ${ }^{*}$ \\ ${ }^{1}$ Department of Obstetrics, Gynecology, and Reproductive Sciences, Division of Maternal-Fetal Medicine, \\ Rutgers Robert Wood Johnson Medical School, New Brunswick, NJ, USA \\ ${ }^{2}$ Princeton Urogynecology, Princeton, NJ, USA \\ ${ }^{3}$ Advanced Tactile Imaging, Inc., Trenton, NJ, USA \\ Email: *egorov@tactile-imaging.com
}

How to cite this paper: Brandt, J.S., Rosen, T., Van Raalte, H., Kurtenos, V. and Egorov, V. (2020) Characterization of Perineum Elasticity and Pubic Bone-Perineal Critical Distance with a Novel Tactile Probe: Results of an Intraobserver Reproducibility Study. Open Journal of Obstetrics and Gynecology, 10, 493-503.

https://doi.org/10.4236/ojog.2020.1040044

Received: March 5, 2020

Accepted: April 6, 2020

Published: April 9, 2020

Copyright ( $\odot 2020$ by author(s) and Scientific Research Publishing Inc. This work is licensed under the Creative Commons Attribution International License (CC BY 4.0).

http://creativecommons.org/licenses/by/4.0/

cc) (i) Open Access

\begin{abstract}
Background: Tactile imaging provides biomechanical mapping of soft tissues. Objective biomechanical and anatomical assessment of critical structures within the vagina and pelvis may allow development and validation of a clinical tool that could assist with clinical decisions regarding obstetrical procedures and mode of delivery. Objective: To assess intraobserver reproducibility of measurements of perineal elasticity and pubic bone-perineal critical distance with a novel tactile probe in pregnant women. Methods: An Antepartum Tactile Imager (ATI) was designed with a vaginal probe resembling a fetal skull. The probe comprises 128 tactile sensors on a double curved surface and measures $46 \mathrm{~mm}$ in width and $72 \mathrm{~mm}$ in length. The probe has a motion tracking sensor that allows acquisition of 3D tactile images. There were two arms of the study. In the first arm, biomechanical mapping of the perineum and pelvic bone location was performed in 10 non-pregnant women for purposes of demonstrating safety and feasibility. In the second arm, biomechanical mapping was performed in 10 pregnant women to explore intraobserver reproducibility. Each subject had two standardized examinations over 3 - 5 minutes by the same observer. Examination comfort and pain levels were assessed by post-procedure survey. Reproducibility was analyzed by intraclass correlation coefficients (ICC) with $95 \%$ confidence intervals and Bland-Altman plots. Bias and the $95 \%$ limits of agreement were also calculated. Results: The safety and feasibility arm of the study demonstrated high degree of safety and tolerability and reliable acquisition of tactile signals. In
\end{abstract}


the reproducibility arm, 10 pregnant women were recruited at mean gestational age of $34.2 \pm 6.5$ weeks. The mean perineum elasticity (Young's modulus, E) was $9.8 \pm 5.9 \mathrm{kPa}$, and the mean pubic bone-perineal critical distance (D) at $20 \mathrm{kPa}$ load was $34.6 \pm 6.2 \mathrm{~mm}$. The ICC was 0.97 [95\% confidence interval (CI) $0.91,0.99$ ] and 0.82 [CI 0.44, 0.95] for E and D respectively, consistent with excellent intrarater agreement. The bias and the $95 \%$ limits of agreement of $\mathrm{E}$ were $-6.3 \%$ and $-29.4 \%$ to $+16.7 \%$, respectively. The bias and the $95 \%$ limits of agreement of $\mathrm{D}$ were $-2.6 \%$ and $-25.3 \%$ to $+20.2 \%$, respectively. Conclusions: The tactile imaging data obtained in the study reproducibly characterized perineal elasticity and pubic bone-perineal critical distance. Further evaluation of this tool in clinical settings is warranted.

\section{Keywords}

Perineal Elasticity, Tactile Imaging, Elastography, Antepartum Predictive Model, Delivery Mode, Perineum, Maternal Birth Trauma

\section{Introduction}

Dramatic changes in the mechanical properties of pelvic tissues take place antepartum to facilitate fetal delivery [1] [2] [3] [4]. Despite this, over $85 \%$ of women suffer obstetric trauma to one or several components of the pelvic floor, including the vagina, perineal body, levator ani muscles, and the anal sphincter complex [5]. Birth injury to these integral structural components leads to pelvic pain and dyspareunia and serves as a potentially modifiable risk factor for subsequent pelvic floor disorders [6] [7]. Unfortunately, there are no effective strategies to prevent perineal damage at the time of delivery beyond elective cesarean delivery, which is associated with potential short-term risks and long-term consequences [8] [9] [10] [11].

Human and animal studies show that several adaptations during pregnancy may reduce potential injury, including increased laxity of pelvic connective tissues and ligaments, increase in genital hiatal area, and alterations in the pelvic floor muscles [1] [2] [3]. Computational models of human parturition demonstrate that mechanical changes in one pelvic component may impact the susceptibility to injury of other pelvic structures [12]. However, the protective effect of pregnancy-induced alterations is variable. Therefore, quantification and knowledge of biomechanical tissue properties of critical pelvic components may allow practical prediction of behavior under applied stress during delivery and obstetrical procedures.

A new device, the Antepartum Tactile Imager (ATI), was designed to characterize perineal elasticity and pubic bone-perineal critical distance. The objective of this study was to assess safety, feasibility, and reproducibility of tactile measurements using this device. In this two-armed study, the feasibility and safety of the ATI was assessed with a cohort of non-pregnant women. Subse- 
quently, an intraobserver reproducibility study was performed in 10 pregnant women.

\section{Materials and Methods}

\section{Study Design}

This study was performed from March 2019 to January 2020. The safety arm was performed in non-pregnant women from March to June 2019, and the reproducibility arm was performed in pregnant women from December 2019 to January 2020. This observational study (clinical trials identifiers NCT03883867) was approved by the Western Institutional Review Board (\#20183400) and the Institutional Review Board of Rutgers Robert Wood Johnson Medical School (\#Pro2018002747). Written informed consent was obtained for each patient prior to study enrollment.

\section{Safety and Feasibility Arm}

The study arm was performed in non-pregnant women as required by the Code of Federal Regulations, Title 45, \$46.204(a). The arm included 10 non-pregnant women who were recruited at a private urogynecology office. Inclusion criteria were adult women, age $21+$ years, with negative urine pregnancy tests and at least one prior vaginal delivery. Exclusion criteria were prior perineal surgery; HIV or hepatitis B positive serology; warty lesions on the vulva; extensive varicose veins on the vulva; active skin infection or ulceration within the vagina/vulva (e.g. Herpes infection); presence of a vaginal septum; and severe hemorrhoids.

During the examination, patients were placed in the dorsal lithotomy position. The ATI probe was covered by a disposable plastic sheath with a water based lubricant. The probe was inserted in the vagina until the sensing surface was internalized, approximately $55 \mathrm{~mm}$. The probe was pushed downward to a maximum pressure load up to $20 \mathrm{kPa}$. Three orthogonal projections of the $3 \mathrm{D}$ vaginal pressure map with real-time ATI probe location was observed by the operator. The complete examination lasted 3 - 5 minutes.

Patients were queried about pain and comfort after the examination. Subjects were assessed about pain using a 4 -point Likert scale with $1=$ no pain, $2=$ mild pain, $3=$ moderate pain, and $4=$ severe pain. The comfort level was assessed using a second 3-point Likert scale with $1=$ more comfortable than manual palpation, 2 = as comfortable as manual palpation, and $3=$ less comfortable than manual palpation.

Reproducibility Arm

The reproducibility arm included 10 pregnant women who were approached at a regional perinatal center. Inclusion criteria were adult women, age $21+$ years, who completed 35+ weeks' gestation, had at least one vaginal delivery, and were planned for a trial of labor. Exclusion criteria were the same in the safety arm of the study. In addition, subjects were excluded in cases of a fetal demise or congenital abnormalities of the fetus.

The tactile examination and the pain and comfort assessments were the same 
as described above. Demographic data, including participants' age, parity, weight, and gestational age were collected. Delivery data, including mode of delivery, perineal laceration, and neonatal birth weight were also collected.

\section{Antepartum Tactile Imager ( $A T I)$}

The ATI was designed as a cart-based device with a medical grade touchscreen computer (Tangent, CA) and a detachable vaginal probe (Figure 1). The ATI probe contains a tactile array with 128 sensors on a double curved surface resembling a fetal skull. The probe's dimensions are $46 \mathrm{~mm}$ in width and $72 \mathrm{~mm}$ in length, curvature radius of $60 \mathrm{~mm}$ in transverse and $102 \mathrm{~mm}$ in sagittal cross-sections, a rectangular sensing area of $26 \mathrm{~mm}$ by $55 \mathrm{~mm}$. The probe has a 6 degree-of-freedom electromagnetic motion tracking sensor (Polhemus, Inc., VT), which allows acquisition of 3D tactile images. The referenced motion sensor was placed under the seat of a custom designed examination chair to eliminate interference for electromagnetic tracking. Biomechanical mapping of the pelvic tissues/structures was performed with attention to the perineum and pubic symphysis location.

Proprietary data processing software was used to calculate perineal elasticity and anatomical distance from the pubic bone and perineal surface, termed the pubic bone-perineal critical distance, at $20 \mathrm{kPa}$ load by the ATI probe. The ATI has quantified perineal elasticity using Young's modulus, which is calculated from spatial gradients in the resulting $3 \mathrm{D}$ tactile images with the use of a non-linear model for the tissue [13]. This approach was validated with multiple pelvic floor models built with two-component silicone (GE Silicones, NY) [14].

Statistical Analysis

Descriptive statistical calculations were performed to ascertain the mean and standard deviations for perineum elasticity (E) and pubic bone-perineal critical distance (D). Intraclass correlation coefficients (ICC) were calculated for these measurements with 95\% confidence intervals (CI) [15]. In addition, the following parameters were calculated as described by Bland and Altman [16]: 1) bias [i.e., the mean of the proportionate difference (the difference between two elasticity measurements divided by the average value of two measurements)]; and 2) 95\% limits of agreement (i.e., 1.96 times the standard deviation of the mean of the proportionate difference). Statistical analysis was performed with STATA version 10.1 (StataCorp LP, TX) and MATLAB version R2018a (MathWorks, MA).

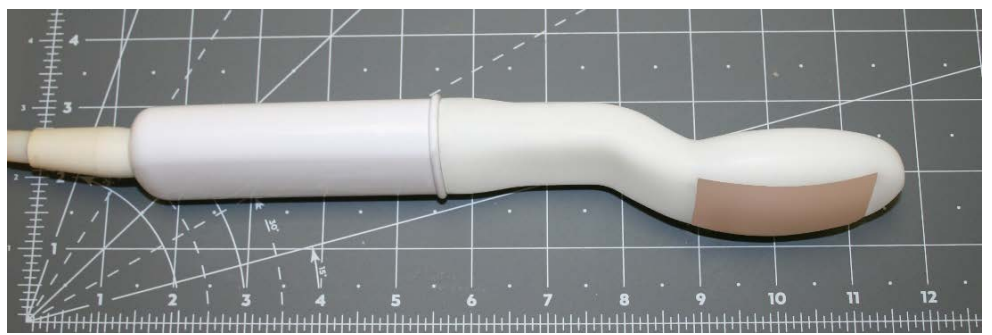

Figure 1. Antepartum tactile imaging probe. 


\section{Results}

\section{Feasibility and Safety Arm}

The study with 10 non-pregnant women demonstrated reliable acquisition of tactile signals and composition of 3D tactile images. The imaging post-processing allowed calculation of $\mathrm{E}$ and $\mathrm{D}$ for each case. The mean $\mathrm{E}$ was $10.4 \pm 2.5 \mathrm{kPa}$, and the mean D was $19.6 \pm 6.1 \mathrm{~mm}$. The pain and comfort assessments revealed a high degree of tolerability. Patients reported that the vaginal tactile imaging was minimally painful (rated $1.7 \pm 0.8$ ) and similar to manual palpation (rated $2.2 \pm 0.8)$. No adverse events were reported.

\section{Reproducibly Arm}

10 pregnant women were recruited for the reproducibility arm. ATI measurements were performed at a mean gestational age of $36.8 \pm 0.6$ weeks. All patients were multiparous and met the study's inclusion criteria. Demographic characteristics and birth outcomes are listed in Table 1. No adverse events were reported.

The mean $\mathrm{E}$ was $9.8 \pm 5.9 \mathrm{kPa}$, and the mean $\mathrm{D}$ was $34.6 \pm 6.2 \mathrm{~mm}$. Figure 2 illustrates the 3 orthogonal projections of the 3D vaginal pressure map with real-time ATI probe location that was captured by the observer in case \#6, which was accompanied by a second degree perineal laceration at vaginal delivery.

The ICC for E was 0.97 [95\% CI 0.91, 0.99], which indicates excellent intrarater agreement. The bias and the $95 \%$ limits of agreement of $\mathrm{E}$ measurement are $-6.3 \%$ and $-29.4 \%$ to $+16.7 \%$. The ICC for D measurement was 0.82 [95\% CI 0.44, 0.95], which also indicates excellent agreement. The bias and $95 \%$ limits of agreement of the D are $-2.6 \%$ and $-25.3 \%$ to $+20.2 \%$. These results are illustrated graphically in Figure 3 and Figure 4.

The pain and comfort assessments revealed a high degree of tolerability. Patients reported that the vaginal tactile imaging was minimally painful (rated 1.7 \pm 0.7 ) and similar to manual palpation (rated $1.9 \pm 0.7$ ).

Table 1. Demographic variables and delivery data for 10 pregnant women in the reproducibility arm.

\begin{tabular}{cc}
\hline Variable & $\mathbf{N}=10$ \\
\hline Age, years & $33.5 \pm 5.3$ \\
Gravida & $3.7 \pm 1.3$ \\
Para & $1.3 \pm 0.5$ \\
Gestational age at exam, weeks & $36.8 \pm 0.6$ \\
Intact perineum & $6(60)$ \\
Laceration & \\
First & $3(30)$ \\
Second & $1(10)$ \\
Third/Fourth & 0 \\
Birthweight, grams & $3274 \pm 276$ \\
Data presented as mean \pm standard deviation and $\mathrm{N}$ (percent).
\end{tabular}




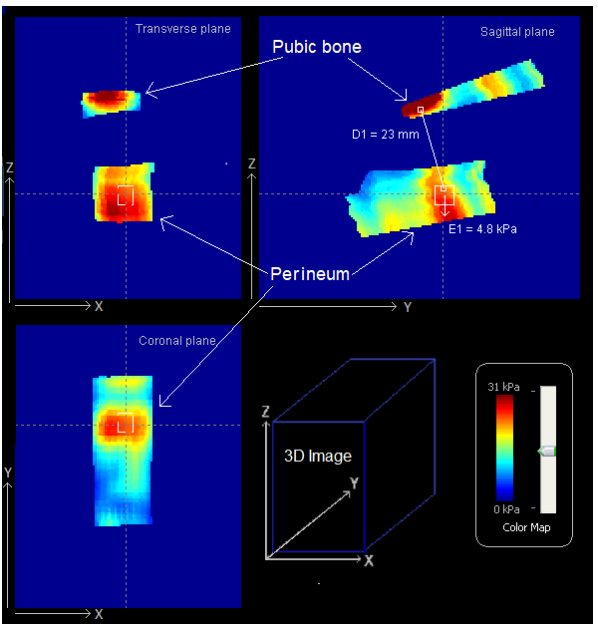

Figure 2. Three-dimensional tactile image of perineum elasticity and pubic bone-perineal critical distance. Three-dimensional tactile image acquired for a 34 -year-old women at 37 weeks' gestation. Attention was given to perineal elasticity and pubic bone-perineal critical distance at a $20 \mathrm{kPa}$ load.

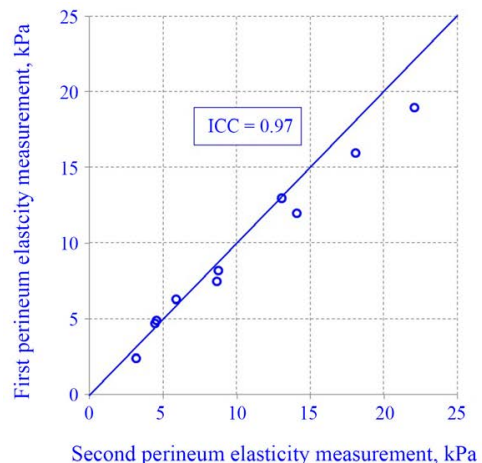

(a)

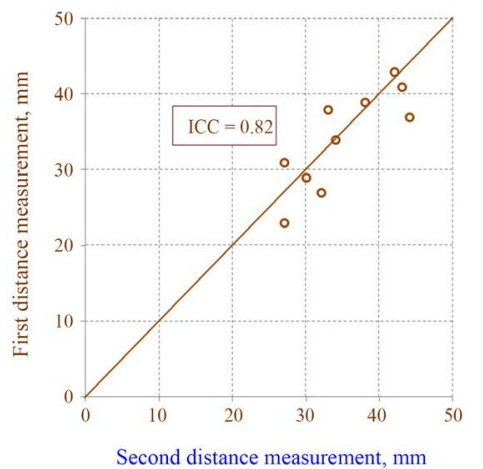

(b)

Figure 3. Intraclass correlation coefficients. Intraclass correlation coefficients (ICC) for two measurements of perineum elasticity (a) and pubic bone-perineal surface distance (b) by the same operator.

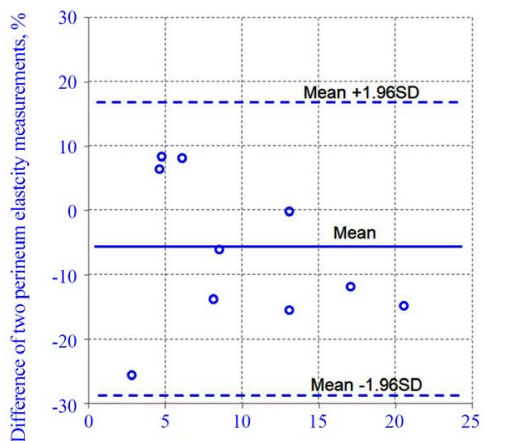

Perineum elasticity (average of two measurements), $\mathrm{kPa}$

(a)

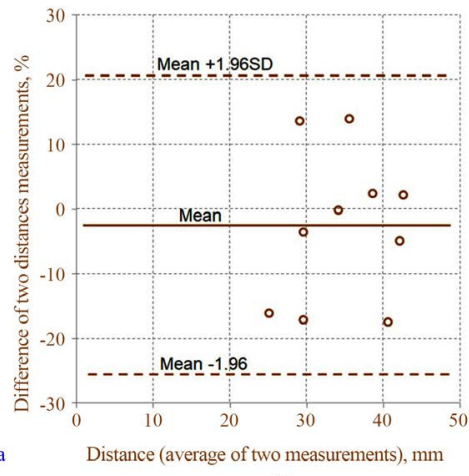

(b)

Figure 4. Bland-Altman scatter plots of difference between two measurements. Bland-Altman scatter plot of the percentage difference between two measurements of perineum elasticity (a) and pubic bone-perineal critical distance (b) by the same operator. The solid lines represent the proportionate mean difference; the dashed lines represent the $95 \%$ limits of agreement. 


\section{Discussion}

\section{Principal Findings}

In this study, we assessed the feasibility, safety, and reproducibility of the ATI, a novel vaginal tactile imager, in a cohort of 10 non-pregnant women and 10 pregnant women. In the feasibility and safety arm, the ATI demonstrated reliable acquisition of tactile signals and composition of 3D tactile images and patients reported pain and comfort assessments consistent with a high degree of tolerability. In the reproducibility arm, the ATI characterized perineum elasticity and pubic bone-perineal critical distance with excellent intrarater agreement.

\section{Results of the Study in Related Context}

In general, elasticity imaging is based on generating stress in the studied tissue in vivo using various static or dynamic means and assessing the resulting strain by ultrasound, MRI [17]-[27] or measurement through tactile imaging [28] [29] [30]. At present, there is no ultrasound probe capable of stress-strain quantitative assessment of the entire birth canal. The ultrasound strain, as well as shear wave elastography, can measure the elasticity in only specifically defined local regions at a time. The results of the current research demonstrate that tactile imaging allows acquisition of 3D stress-strain data and elasticity assessment of the perineum.

Despite the obvious fact that vaginal delivery is a biomechanical process, currently there are no tools in clinical practice that can measure the biomechanical properties of pelvic tissue in women before delivery [31] [32] [33] [34]. We previously reported our experience with the Vaginal Tactile Imager (VTI), which was developed as a biomechanical mapping device to assess vaginal and pelvic floor conditions [30]. The VTI vaginal probe has an elongated linear design with a 96 linear sensor array on both sides of the probe [35] to allow acquisition of pressure patterns along the vaginal walls, but it does not have a $3 \mathrm{D}$ motion tracking system and has. The ATI has 3D imaging capability and double convex head with $2 \mathrm{D}$ tactile sensor array to provide stress distribution to the perineum during the ATI measurement similar to the stress distribution during the delivery.

Multiple computational models of vaginal birth have been developed [3] [12] [36] [37] [38] [39] [40]. While these models provide insight regarding the biomechanics of human parturition, they are limited by numerous assumptions, as mechanical tissue properties in late-pregnancy remain unknown. These models are often based on the data collected with cadaver tissues with the lack of muscle and tissue tone that, as a result, represent a potential source of significant inaccuracy in modeling in vivo deformation [41] [42] [43]. Some of these models used simplified boundary conditions and did not always consider the mechanical interaction between the fetal head and maternal pelvic tissues. Thus, to date, we cannot find a validated in vivo biomechanical model of vaginal delivery which could predict personalized delivery outcomes. The results of this study (and future planned exploration) may provide some of that missing data to be used in 
these computational models.

\section{Research and Clinical Implications}

This study is in the first exploratory stage of the project. A finite element model development and a validation study will follow to establish nomograms for perineum elasticity and pubic bone-perineal critical distance and to establish clinically significant cut-points to guide clinical care and decision making. This new system may open a new technical capability in women's health and change the established clinical practice. This approach may offer reliable causations between pelvic floor conditions, child size/weight on one side and delivery modes/delivery procedures and involved risk of injuries and their consequences on the other side.

\section{Strengths and Limitations}

The strength of this study lies in the novel approach for perineum characterization. The perineal elasticity receives quantification in terms of Young's modulus from stress-strain data. Additionally, pubic bone-perineal critical distance was quantified. This distance predicts capability of the delivery canal to pass through the fetus at predefined level of strain. Both of these measures may be associated with perineal lacerations, and the results of our study justify further prospective evaluation in clinical practice.

The study has some limitations. While the study evaluated the intrarater agreement of ATI measurements, interrater reproducibility was not evaluated. This will be performed in future studies of the ATI. In addition, the study had a small sample size with limited power to evaluate the association between perineum elasticity and pubic bone-perineal critical distance on perineal lacerations. The intention of this study, however, was not to assess clinical outcomes, but rather to assess safety, feasibility, and reproducibility of the ATI rather than efficacy.

\section{Conclusion}

In this study, the safety, feasibility, and reproducibility of a novel device were evaluated. The results of this study demonstrated that the new vaginal probe was well tolerated by study participants and produced reproducible characterization of perineum elasticity and pubic bone-perineal critical distance, both factors that may be associated with perineal lacerations and pelvic floor injury. Further evaluation of the ATI in clinical settings is warranted.

\section{Acknowledgements}

The study was supported by research grant R43HD095223 from Eunice Kennedy Shriver National Institute of Child Health \& Human Development (NICHD), USA. The content is solely the responsibility of the authors and does not necessarily represent the official views of the National Institutes of Health.

We would like to acknowledge the contributions of our clinical research coordinators, Shama Khan, Jessica Sowden, and Alison Shaltis. 


\section{Conflicts of Interest}

The authors have no conflicts of interest. Dr. Egorov is a co-founder of Advanced Tactile Imaging, Inc.

\section{References}

[1] Alperin, M. (2016) Impact of Pregnancy and Delivery on Pelvic Floor Biomechanics. In: Hoyte, L. and Damaser, M.S., Eds., Biomechanics of the Female Pelvic Floor, Elsevier, Amsterdam, 229-238. https://doi.org/10.1016/B978-0-12-803228-2.00010-6

[2] Betschart, C. and Wisser, J. (2017) The Effect of Pregnancy on the Pelvic Floor. In: Doumouchtsis, S.K., Ed., Childbirth Trauma, Springer-Verlag, London, 57-68. https://doi.org/10.1007/978-1-4471-6711-2_4

[3] Lien, K.C., Mooney, B., DeLancey, J.O. and Ashton-Miller, J.A. (2004) Levator Ani Muscle Stretch Induced by Simulated Vaginal Birth. Obstetrics \& Gynecology, 103, 31-40. https://doi.org/10.1097/01.AOG.0000109207.22354.65

[4] Svabík, K., Shek, K.L. and Dietz, H.P. (2009) How Much Does the Levator Hiatus Have to Stretch during Childbirth? BJOG: An International Journal of Obstetrics \& Gynaecology, 116, 1657-1662. https://doi.org/10.1111/j.1471-0528.2009.02321.x

[5] Kettle, C. and Tohill, S. (2011) Perineal Care. BMJ Clinical Evidence, 4, 1401.

[6] Hallock, J.L. and Handa, V.L. (2016) The Epidemiology of Pelvic Floor Disorders and Childbirth: An Update. Obstetrics and Gynecology Clinics of North America, 43, 1-13. https://doi.org/10.1016/j.ogc.2015.10.008

[7] Quiroz, L.H. and Shobeiri, A.S. (2017) Epidemiology of Childbirth Trauma and Associated Pelvic Floor Disorders. In: Doumouchtsis, S.K., Ed., Childbirth Trauma, Springer-Verlag, London, 57-68. https://doi.org/10.1007/978-1-4471-6711-2_3

[8] Cunningham, F.G., Leveno, K.J., Bloom, S.L., Spong, C.Y., Dashe, J.S., Hoffman, B.L., Casey, B.M. and Sheffield, J.S. (2014) Williams Obstetrics. 24th Edition, McGraw-Hill Education, New York, 1-1376.

[9] Gauthaman, N. and Doumouchtsis, S.K. (2017) Mode of Delivery and Perineal Trauma. In: Doumouchtsis, S.K., Ed., Childbirth Trauma, Springer-Verlag, London, 57-68. https://doi.org/10.1007/978-1-4471-6711-2_5

[10] Boyle, A., Reddy, U.M., Landy, H.J., et al. (2013) Primary Cesarean Delivery in the United States. Obstetrics \& Gynecology, 122, 33-40. https://doi.org/10.1097/AOG.0b013e3182952242

[11] American College of Obstetricians and Gynecologists; Society for Maternal-Fetal Medicine (2014) Obstetric Care Consensus No. 1: Safe Prevention of the Primary Cesarean Delivery. Obstetrics \& Gynecology, 123, 693-711. https://doi.org/10.1097/01.AOG.0000444441.04111.1d

[12] Jing, D., Ashton-Miller, J.A. and DeLancey, J.O. (2012) A Subject-Specific Anisotropic Visco-Hyperelastic Finite Element Model of Female Pelvic Floor Stress and Strain during the Second Stage of Labor. Journal of Biomechanics, 45, 455-460. https://doi.org/10.1016/j.jbiomech.2011.12.002

[13] Egorov, V., van Raalte, H. and Lucente, V. (2012) Quantifying Vaginal Tissue Elasticity under Normal and Prolapse Conditions by Tactile Imaging. International Urogynecology Journal, 23, 459-466. https://doi.org/10.1007/s00192-011-1592-Z

[14] Egorov, V., Tsyuryupa, S., Kanilo, S., Kogit, M. and Sarvazyan, A. (2008) Soft Tissue Elastometer. Medical Engineering \& Physics, 30, 206-212. https://doi.org/10.1016/j.medengphy.2007.02.007

[15] Bartlett, J.W. and Frost, C. (2008) Reliability, Repeatability and Reproducibility: 
Analysis of Measurement Errors in Continuous Variables. Ultrasound Obstetrics \& Gynecology, 31, 466-475. https://doi.org/10.1002/uog.5256

[16] Bland, J.M. and Altman, D.G. (2003) Applying the Right Statistics: Analyses of Measurement Studies. Ultrasound Obstetrics \& Gynecology, 22, 85-93.

https://doi.org/10.1002/uog.122

[17] Parker, K.J., Huang, S.R., Musulin, R.A. and Lerner, R.M. (1990) Tissue Response to Mechanical Vibrations for "Sonoelasticity Imaging". Ultrasound in Medicine \& Biology, 16, 241-246. https://doi.org/10.1016/0301-5629(90)90003-U

[18] Ophir, J., Cespedes, I., Ponnekanti, H., Yazdi, Y. and Li, X. (1991) Elastography: A Quantitative Method for Imaging the Elasticity of Biological Tissues. Ultrasonic Imaging, 13, 111-134. https://doi.org/10.1177/016173469101300201

[19] Sarvazyan, A.P., Skovoroda, A.R., et al. (1995) Biophysical Bases of Elasticity Imaging. Acoustical Imaging, 21, 223-240. https://doi.org/10.1007/978-1-4615-1943-0_23

[20] Sarvazyan, A.P., Rudenko, O.V., Swanson, S.D., Fowlkes, J.B. and Emelianov, S.Y. (1998) Shear Wave Elasticity Imaging: A New Ultrasonic Technology of Medical Diagnostics. Ultrasound in Medicine \& Biology, 24, 1419-1435. https://doi.org/10.1016/S0301-5629(98)00110-0

[21] Manduca, A., Oliphant, T.E., et al. (2001) Magnetic Resonance Elastography: Non-Invasive Mapping of Tissue Elasticity. Medical Image Analysis, 5, 237-254. https://doi.org/10.1016/S1361-8415(00)00039-6

[22] Nightingale, K., Soo, M.S., Nightingale, R. and Trahey, G. (2002) Acoustic Radiation Force Impulse Imaging: In Vivo Demonstration of Clinical Feasibility. Ultrasound in Medicine \& Biology, 28, 227-235. https://doi.org/10.1016/S0301-5629(01)00499-9

[23] Greenleaf, J.F., Fatemi, M. and Insana, M. (2003) Selected Methods for Imaging Elastic Properties of Biological Tissues. Annual Review of Biomedical Engineering, 5, 57-78. https://doi.org/10.1146/annurev.bioeng.5.040202.121623

[24] Giovannini, M., Hookey, L.C., Bories, E., Pesenti, C., Monges, G. and Delpero, J.R. (2006) Endoscopic Ultrasound Elastography: The First Step towards Virtual Biopsy? Preliminary Results in 49 Patients. Endoscopy, 38, 344-348. https://doi.org/10.1055/s-2006-925158

[25] Kiss, M.Z., Hobson, M.A., Varghese, T., Harter, J., Kliewer, M.A., Hartenbach, E.M. and Zagzebski, J.A. (2006) Frequency-Dependent Complex Modulus of the Uterus: Preliminary Results. Physics in Medicine \& Biology, 51, 3683-3695. https://doi.org/10.1088/0031-9155/51/15/006

[26] Bensamoun, S.F., Ringleb, S.I., Littrell, L., Chen, Q., Brennan, M., Ehman, R.L. and An, K.N. (2006) Determination of Thigh Muscle Stiffness Using Magnetic Resonance Elastography. Journal of Magnetic Resonance Imaging, 23, 242-247. https://doi.org/10.1002/jmri.20487

[27] Wells, P.N.T. (2011) Medical Ultrasound: Imaging of Soft Tissue Strain and Elasticity. Journal of the Royal Society, Interface, 8, 1521-1549.

https://doi.org/10.1098/rsif.2011.0054

[28] Egorov, V. and Sarvazyan, A.P. (2008) Mechanical Imaging of the Breast. IEEE Trans Med Imaging, 27, 1275-1287. https://doi.org/10.1109/TMI.2008.922192

[29] Weiss, R.E., Egorov, V., Ayrapetyan, S., Sarvazyan, N. and Sarvazyan, A. (2008) Prostate Mechanical Imaging: A New Method for Prostate Assessment. Urology, 71, 425-429. https://doi.org/10.1016/j.urology.2007.11.021

[30] Egorov, V, van Raalte, H., Lucente, V. and Sarvazyan, A. (2016) Biomechanical 
Characterization of the Pelvic Floor Using Tactile Imaging. In: Hoyte, L. and Damaser, M.S., Eds., Biomechanics of the Female Pelvic Floor, Elsevier, Amsterdam, 317-348. https://doi.org/10.1016/B978-0-12-803228-2.00016-7

[31] Ashton-Miller, J.A. and Delancey, J.O. (2009) On the Biomechanics of Vaginal Birth and Common Sequelae. Annual Review of Biomedical Engineering, 11, 163-176. https://doi.org/10.1146/annurev-bioeng-061008-124823

[32] Parente, M.P.L. (2008) Biomechanics of the Pelvic Floor during Vaginal Delivery. PhD Thesis, Faculty of Engineering, University of Porto, Portugal, 1-254.

[33] Jansova, M., Kalis, V., Rusavy, Z., Zemcik, R., Lobovsky, L. and Laine, K. (2014) Modeling Manual Perineal Protection during Vaginal Delivery. International Urogynecology Journal, 25, 65-71. https://doi.org/10.1007/s00192-013-2164-1

[34] Zemčík, R., Karbanova, J., Kalis, V., Lobovský, L., Jansová, M. and Rusavy, Z. (2012) Stereophotogrammetryof the Perineum during Vaginal Delivery. International Journal of Gynecology \& Obstetrics, 119, 76-80. https://doi.org/10.1016/j.ijgo.2012.05.018

[35] Egorov, V., Shobeiri, A.S., Takacs, P., Hoyte, L., Lucente, V. and van Raalte, H. (2018) Biomechanical Mapping of the Female Pelvic Floor: Prolapse versus Normal Conditions. Open Journal of Obstetrics and Gynecology, 8, 900-925. https://doi.org/10.4236/ojog.2018.810093

[36] D’Aulignac, D., Martins, J.A.C., Pires, E.B., Mascarenhas, T. and Jorge, R.M.N. (2005) A Shell Finite Element Model of the Pelvic Floor Muscles. Computer Methods in Biomechanics and Biomedical Engineering, 8, 339-347. https://doi.org/10.1080/10255840500405378

[37] Martins, J.A.C., Pato, M.P.M., Pires, E.B., Jorge, R.M.N., Parente, M. and Mascarenhas, T. (2007) Finite Element Studies of the Deformation of the Pelvic Floor. Annals of New York Academy of Science, 1101, 316-334. https://doi.org/10.1196/annals.1389.019

[38] Noakes, K.F., Pullan, A.J., Bissett, I.P. and Cheng, L.K. (2008) Subject Specific Finite Elasticity Simulations of the Pelvic Floor. Journal of Biomechanics, 41, 3060-3065. https://doi.org/10.1016/j.jbiomech.2008.06.037

[39] Hoyte, L., Damaser, M.S., Warfield, S.K., Chukkapalli, G., Majumdar, A., Choi, D.J., Trivedi, A. and Krysl, P. (2008) Quantity and Distribution of Levator Ani Stretch during Simulated Vaginal Childbirth. American Journal of Obstetrics and Gynecology, 199, 198.e1-198.e5. https://doi.org/10.1016/j.ajog.2008.04.027

[40] Parente, M.P., Natal Jorge, R.M., Mascarenhas, T., Fernandes, A.A. and Silva-Filho, A.L. (2010) Computational Modeling Approach to Study the Effects of Fetal Head Flexion during Vaginal Delivery. American Journal of Obstetrics \& Gynecology, 203, 217.e1-217.e 6. https://doi.org/10.1016/j.ajog.2010.03.038

[41] Rahn, D.D., Ruff, M.D., Brown, S.A., Tibbals, H.F. and Word, R.A. (2008) Biomechanical Properties of the Vaginal Wall: Effect of Pregnancy, Elastic Fiber Deficiency, and Pelvic Organ Prolapse. American Journal of Obstetrics \& Gynecology, 198, 590.e1-590.e 6. https://doi.org/10.1016/j.ajog.2008.02.022

[42] Prantil, R.L., Jankowski, R.J., Kaiho, Y., de Groat, W.C., Chancellor, M.B., Yoshimura, N. and Vorp, D.A. (2007) Ex Vivo Biomechanical Properties of the Female Urethra in a Rat Model of Birth Trauma. American Journal of Physiology-Renal Physiology, 292, F1229-F1237. https://doi.org/10.1152/ajprenal.00292.2006

[43] Rubod, C., Boukerrou, M., Brieu, M., Jean-Charles, C., Dubois, P. and Cosson, M. (2008) Biomechanical Properties of Vaginal Tissue: Preliminary Results. International Urogynecology Journal, 19, 811-816. https://doi.org/10.1007/s00192-007-0533-3 